Received: 03.07 .2018

Revised: 30.07 .2018

Accepted: 17.08 .2018

DOI: $10.17804 / 2410-9908.2018 .4 .034-041$

\title{
EFFECT OF HEAT TREATMENT ON THE STATE OF INTERLAYER INTERFACES AND MAGNETORESISTIVE PROPERTIES OF $\mathrm{CO}_{90} \mathrm{Fe}_{10} / \mathrm{Cu}$ SUPERLATTICES
}

\author{
S. A. Chuprakov ${ }^{\text {a) }}$, I. V. Blinov ${ }^{\text {b) }}$, M. A. Milyaev ${ }^{\text {c) }}$ and V. V. Popov ${ }^{\text {d) }}$ \\ M.N. Miheev Institute of Metal Physics, Ural Branch of RAS, \\ 18 S. Kovalevskoy St., Ekaterinburg, 620108, Russian Federation \\ a) (iD http://orcid.org/0000-0002-1026-6203; 《chuprakov@imp.uran.ru; \\ b) (ID http://orcid.org/0000-0002-4126-9351; $\otimes$ blinov@imp.uran.ru; \\ c) (ID http://orcid.org/0000-0001-7828-3571; milyaev@imp.uran.ru; \\ d) (iD http://orcid.org/0000-0002-9291-0102; 《 vpopov@imp.uran.ru \\ *Corresponding author. E-mail: chuprakov@imp.uran.ru \\ Address for correspondence: 18, ul. S. Kovalevskoy, 620990, Ekaterinburg, Russian Federation \\ Tel.: +7 (343) 37442 14; fax: +7 (343) 3745244
}

The effect of the structure of interfaces on the magnetoresistance properties of $\mathrm{CoFe} / \mathrm{Cu}$ superlattices has been studied by the method of nuclear magnetic resonance (NMR). It was found that, with increasing annealing temperature, the number of Co atoms involved in the formation of interlayer boundaries increases and the fraction of highly perfect interfaces decreases. It is demonstrated that the deteriorated state of interlayer boundaries results in a decrease in GMR.

Keywords: giant magnetoresistance, superlattices, interfaces, nuclear magnetic resonance

\section{Acknowledgments}

The X-ray analysis was performed on the Empyrean PANalytical Series 2 diffractometer installed at the collective use center of IPM UB RAS.

The work was performed within the frame of the state assignment on the Spin subject, No. AAAA-A18-118020290104-2, and UB RAS project No. 8-10-2-37.

\section{References}

1. Wetzig K., Schneider C.M. Metal Based Thin Films for Electronics. WILEYVCH GmbH \& Co. KGaA, Weinheim, 2003, 388 p. DOI: 10.1002/3527602534.

2. Mallinson J.C. Magnetoresistive and Spin Valve Heads. Fundamentals and Applications, second edition, ACADEMIC PRESS, San Diego, 2002, 179 p.

3. Kataoka N., Saito K., Fujimori H. Magnetoresistance of Co-X/Cu multilayers. J. Magn. Mater., 1993. vol. 121, pp. 383-385. DOI: 10.1016/0304-8853(93)91226-W.

4. Saito Y., Hashimoto S., Inomata K. Giant magnetoresistance in $\mathrm{Co} / \mathrm{Cu}, \mathrm{Co} 9 \mathrm{Fe} / \mathrm{Cu}$, and Co7.5Fe2.5/Cu multilayers. IEEE Transactions on Magnetics, 1992, vol. 28, no. 23, pp. 2751-2753. DOI: $10.1109 / 20.179617$

5. Wang D., Anderson J., Daughton J.M. Thermally table, low saturation field, low hysteresis, high GMR CoFe/Cu multilayers. IEEE Transactions on Magnetics, 1997, vol. 33, no. 5, pp. 3520-3522. DOI: $10.1109 / 20.619484$.

6. Kuch W., Marley A.C., Parkin S.S.P. Seeded epitaxy of Co90Fe10/Cu multilayers on $\mathrm{MgO}(001)$ :Influence of Fe seed layer thickness. J. Appl. Phys., 1998, vol. 83, no. 9, pp. 4709-4713. DOI:10.1063/1.367259. 
7. Kano H., Iwasaki Y., Hayashi K., Aso K. Magnetoresistance of Co_X/Cu (X = Fe, Ni) multilayers. J. Magn. Magn. Mater., 1993, vol. 126, pp. 445-447. DOI:10.1016/0304-8853(93)90651-H.

8. Ustinov V.V., Milyaev M.A., Naumova L.I. Giant Magnetoresistance of Metallic ExchangeCoupled Multilayers and Spin Valves. Physics of Metals and Metallography, 2017, vol. 118, pp. 1300-1359. DOI: 10.1134/S0031918X17130038.

9. Thomson T., Riedi P.C., Greig D. Interfacial quality and giant magnetoresistance in MBE-grown $\mathrm{Co} / \mathrm{Cu}(111)$ superlattices. Phys. Rev. B, 1994, vol. 50, no. 14, pp. 10319-10322. DOI: 10.1103/PhysRevB.50.10319.

10. Thomson T., Riedi P.C., Wellock K.P., Hickey B.J. ${ }^{59}$ Co nuclear magnetic resonance studies of interfacial defects in molecular beam epitaxy grown $\mathrm{Co} / \mathrm{Cu}(111)$ multilayer. J. Appl. Phys., 1995, vol. 81, no. 8, pp. 4469-4471. DOI: 10.1063/1.364980.

11. Chuprakov S.A., Krinitsin T.P., Bannikova N.S., Blinov I.V., Verkhovskii S.V., Milyaev M.A., Popov V.V., Ustinov V.V. Interface Structure and Magnetoresistance Studies of $[\mathrm{Co} / \mathrm{Cu}] \mathrm{n}$ Superlattices by Means of NMR and TEM. Solid State Phenomena, 2014, vol. 215, pp. 358-364. DOI: 10.4028/www.scientific.net/SSP.215.358.1.

12. Chuprakov S.A., Bannikova N.S., Blinov I.V., Krinitsina T.P., Milyaev M.A., Popov V.V., Ustinov V.V. Investigation of interfaces of multilayer $\mathrm{Co} / \mathrm{Cu}$ structures using the method of nuclear magnetic resonance. Physics of Metals and Metallography, 2015, vol. 116, pp. 136-140. DOI: $10.1134 / \mathrm{S} 0031918 X 15020040$.

13. Chuprakov S.A., Bannikova N.S., Blinov I.V., Krinitsina T.P., Milyaev M.A., Popov V.V., Ustinov V.V. Study of the structure of interlayer boundaries in $[\mathrm{Co} / \mathrm{Cu}] 10$ superlattices by methods of NMR and X-ray reflectometry. Physics of Metals and Metallography, 2016, vol. 117, pp. 1192-1197. DOI: $10.1134 /$ S0031918X16120048.

14. Thomson T., Riedi P.C. NMR Studies of Sputtered CoFe Alloy Thin Films. IEEE Transactions on Magnetics, 1998, vol. 34, no. 4, pp. 1045-1047. DOI: 10.1109/20.706352. 
Подана в журнал: 03.07.2018

УДК 539.216.2:543.429.3

DOI: $10.17804 / 2410-9908.2018 .4 .034-041$

\title{
ВЛИЯНИЕ ТЕРМООБРАБОТКИ НА СОСТОЯНИЕ МЕЖСЛОЙНЫХ ГРАНИЦ И МАГНИТОРЕЗИСТИВНЫЕ ХАРАКТЕРИСТИКИ В СВЕРХРЕШЕТКАХ $\mathrm{CO}_{90} \mathrm{Fe}_{10} / \mathrm{Cu}$
}

\author{
С. А. Чупраков ${ }^{\text {a** }}$, И. В. Блинов ${ }^{\text {() }}$, М. А. Миляев ${ }^{\text {в) }}$ В. В. Попов ${ }^{\text {г) }}$ \\ Федеральное государственное бюджетное учреждение науки \\ Институт физики металлов имени М.Н. Михеева Уральского отделения Российской академии наук, \\ ул. С. Ковалевской, 18, Екатеринбург, Российская Федераиция \\ a) (D) http://orcid.org/0000-0002-1026-6203; هchuprakov@imp.uran.ru; \\ б) (iD http://orcid.org/0000-0002-4126-9351; هblinov@imp.uran.ru; \\ в) (ID http://orcid.org/0000-0001-7828-3571; milyaev@imp.uran.ru;

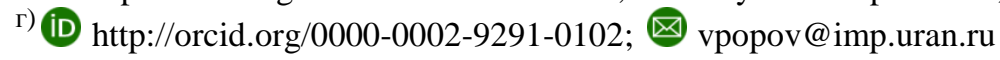

\begin{abstract}
"Ответственный автор. Электронная почта: chuprakov@imp.uran.ru Адрес для переписки: ул. С. Ковалевской, 18, 620990, Екатеринбург, Российская Федерация
\end{abstract} \\ Тел.: +7 (343) 374-42-14; факс: +7 (343) 374-52-44
}

Методом ядерного магнитного резонанса (ЯМР) исследовано влияние структуры интерфейсов на магниторезистивные свойства сверхрешеток $\mathrm{CoFe} / \mathrm{Cu}$. Установлено, что при увеличении температуры отжига возрастает число атомов кобальта, участвующих в формировании межслойных границ и уменьшается доля высокосовершенных интерфейсов. Показано, что ухудшение состояния границ приводит к уменьшению величины эффекта гигантского магнитосопротивления.

Ключевые слова: гигантское магнитосопротивление, сверхрешетки, интерфейсы, ядерный магнитный резонанс.

\section{1. Введение}

Многослойные обменносвязанные металлические магнитные сверхрешетки, обладающие эффектом гигантского магнитосопротивления (ГМС), находят широкое примерение в устройствах спинтроники и радиоэлектроники $[1,2]$. Одними из часто используемых магнитных материалов, слои которого входят в состав магнитных сверхрешеток, являются сплавы $\mathrm{Co}_{1-\mathrm{x}} \mathrm{Fe}_{\mathrm{x}}$, используемые совместно, например, со слоями $\mathrm{Cu}$. Ранее было установлено, что замена в системе $\mathrm{Co} / \mathrm{Cu}$ кобальта на сплав $\mathrm{Co}_{90} \mathrm{Fe}_{10}$ приводит к увеличению эффективного магнитного момента на атом [3], повышению магнитосопротивления и уменьшению магнитного гистерезиса [3, 4], а также к улучшению термической стабильности [5].

При изменении толщины слоев меди в сверхрешетках $\mathrm{Co}_{1-\mathrm{x}} \mathrm{Fe}_{\mathrm{x}} / \mathrm{Cu}$ наблюдается осциллирующий характер изменения межслойного обменного взаимодействия с периодом осцилляций около $12 \AA$, сопровождающийся периодическим изменением величины ГМС [4].

Ранее наибольшие для системы $\mathrm{CoFe} / \mathrm{Cu}$ значения магнитосопротивления $63 \%$ и 55 \% были получены, соответственно, в работах [6] и [7] при толщинах слоев меди $t_{\mathrm{Cu}}=9 \AA$ и $t_{\mathrm{Cu}}=10 \AA$, что соответствует первому максимуму антиферромагнитного межслойного обменного взаимодействия. При этом поле магнитного насыщения составляло $H_{\mathrm{s}}=3-5$ кЭ. Недавно авторами работы [8] были получены рекордные значения магнитосопротивления $81 \%$ при комнатной температуре в сверхрешетках $\left.\left[\mathrm{Co}_{90} \mathrm{Fe}_{10}(1,5 \mathrm{Hм}) / \mathrm{Cu} 0,95 \mathrm{Hм}\right)\right]_{24}$.

Известно, что магнитные и магнитотранспортные свойства зависят от структурных особенностей слоев и межслойных границ - интерфейсов, формируемых в процесее приготовле- 
ния $[9,10]$. Для исследования состояния межслойных границ и структурных особенностей слоев успешно применяется метод ядерного магнитного резонанса [11-13].

Основная идея использования метода ЯМР при исследовании многослойных структур $\mathrm{Co} / \mathrm{Cu}$ заключается в следующем. В результате сверхтонкого взаимодействия магнитные моменты атомов кобальта создают в месте расположения ядер ${ }^{59}$ Со локальные магнитные поля, величина и направление которых существенно зависят от магнитных и структурных особенностей ближайшего окружения ядра-зонда. Метод ЯМР позволяет исследовать распределение этих локальных полей в образце, а потому является мощным методом изучения структурных характеристик многослойных наноструктур.

В настоящей работе поставлена задача, исследовать структуру межслойных границ в сверхрешетках $\mathrm{CoFe} / \mathrm{Cu}$ и влияние состояния границ на магниторезистивные характеристики этих сверхрешеток. Состоянние интерфейсов изменялось путем отжига образцов при различных температурах $\left(400,450{ }^{\circ} \mathrm{C}\right)$, длительность отжига 1 ч.

\section{2. Материал и методика}

Образцы были приготовлены в напылительной системе Ulvac MPS-4000-C6 методом магнетронного напыления при постоянном токе на стеклянные подложки (Corning). Поверхность подложки перед напылением очищали ионным травлением в атмосфере аргона в напылительной системе. Материал защитного слоя - Та толщиной 5 нм. В качестве буферного слоя использовали композитный буферный слой: $\mathrm{Ta}(5 \mathrm{Hм}) / \operatorname{PyCr}(5$ нм). Парамагнитный сплав пермаллой-хром (PуCr) состава $\left(\mathrm{Ni}_{80} \mathrm{Fe}_{20}\right)_{60} \mathrm{Cr}_{40}$.

Напыление сверхрешеток выполнялось при комнатной температуре подложки, при фиксированном давлении аргона 0,1 Па и мощности магнетронов 100 Вт. Структурная формула образцов имеет следующий вид: Стекло//Тa(5нм)/PyCr(5нм)/[Cog0 $\mathrm{Fe}_{10}(1,6 \mathrm{Hm}) / \mathrm{Cu}(0,95 \mathrm{Hm}]_{12} / \mathrm{Ta}(5 \mathrm{Hм})$. В дальнейшем приготовленные образцы были отожжены при температурах $T_{\text {отж}}=400,450{ }^{\circ} \mathrm{C}$ при давлении $P=10^{-5}$ Па в течение 1 ч.

Рентгенографические исследования выполнены при использовании программноаппаратного комплекса Philips Empyrean в излучении $\mathrm{Cu} K_{\alpha}$. Методом ядерного магнитного резонанса на ядрах ${ }^{59}$ Со были получены спектры исследуемых образцов в диапазоне частот 130-260 МГц. Регистрация спектров была выполнена на импульсном спектрометре ЯМР с использованием стандартной методики спинового эха при температуре жидкого гелия $(4,2 \mathrm{~K})$ в локальном магнитном поле - внешнее магнитное поле отсутствовало. Измерительная катушка изготовлена из медной посеребренной проволоки. Катушка представляет собой соленоид, в который помещается образец - пластина $10 \times 10$ мм. В этой геометрии сигнал ЯМР возбуждается от областей образца, спонтанный магнитный момент которых перпендикулярен плоскости пленки. Сигнал спинового эха $E\left(2 t_{\mathrm{del}}\right)$ формируется последовательностью двух когерентных радиочастотных импульсов $\left(\tau_{p}\right)_{x}-t_{\mathrm{del}}-\left(\tau_{p}\right)_{y}-t_{\mathrm{del}}-\mathrm{Echo}$, создающих в резонансной катушке с образцом переменное магнитное поле с амплитудой круговой компоненты $H_{1} \approx 10$ Э. Длительность импульсов $\tau_{p}$ составляла 0,5 мкс, временной промежуток между импульсами $t_{\mathrm{del}}-11$ мкс. Шаг изменения частоты составлял 1 МГц.

Интенсивности $I(v)=E\left(t_{\mathrm{del}}=0\right)$ были определены из измерений скорости затухания сигнала спинового эха на различных участках спектра. Постоянство добротности катушки ЯМР, импеданса резонансного контура и уровень $H_{1}$ контролировались по ширине переднего фронта и амплитуды радиочастотного импульса в каждой точке спектра.

Коэффициент усиления РЧ-поля (ๆ) в магнитоупорядоченном состоянии пленки был определен для каждой линии спектра. Обнаружено, что величина з остается практически неизменной: $\frac{\Delta \eta}{\eta} \leq 0,1$. Соответствующие поправки $\Delta Q, \Delta \eta$ и $T_{2}$ были использованы при расчете интенсивности линий спектра.

Магнитосопротивление было измерено по стандартной методике четырехконтактным методом на постоянном токе при протекании тока в плоскости слоев. Все измерения 
выполнены при комнатной температуре. Магнитосопротивление определяли по формуле: $M R=\left(R(H)-R_{\mathrm{s}}\right) / R_{\mathrm{s}}$, где $R_{\mathrm{s}}-$ сопротивление в поле магнитного насыщения.

\section{3. Результаты и обсуждение}

Проведенные исследования показали, что в исследуемых сверхрешетках $\mathrm{CoFe} / \mathrm{Cu}$ после напыления максимальная величина магнитосопротивления составляет $\Delta R / R_{\mathrm{s}}=62,5 \%$, поле магнитного насыщения $H_{\mathrm{s}}=10$ кЭ. При увеличении температуры отжига от 400 до $450{ }^{\circ} \mathrm{C}$ магнитосопротивление уменьшается от 22 до 0,3 \% соответственно. Соответствующие данные представлены на рис. 1.

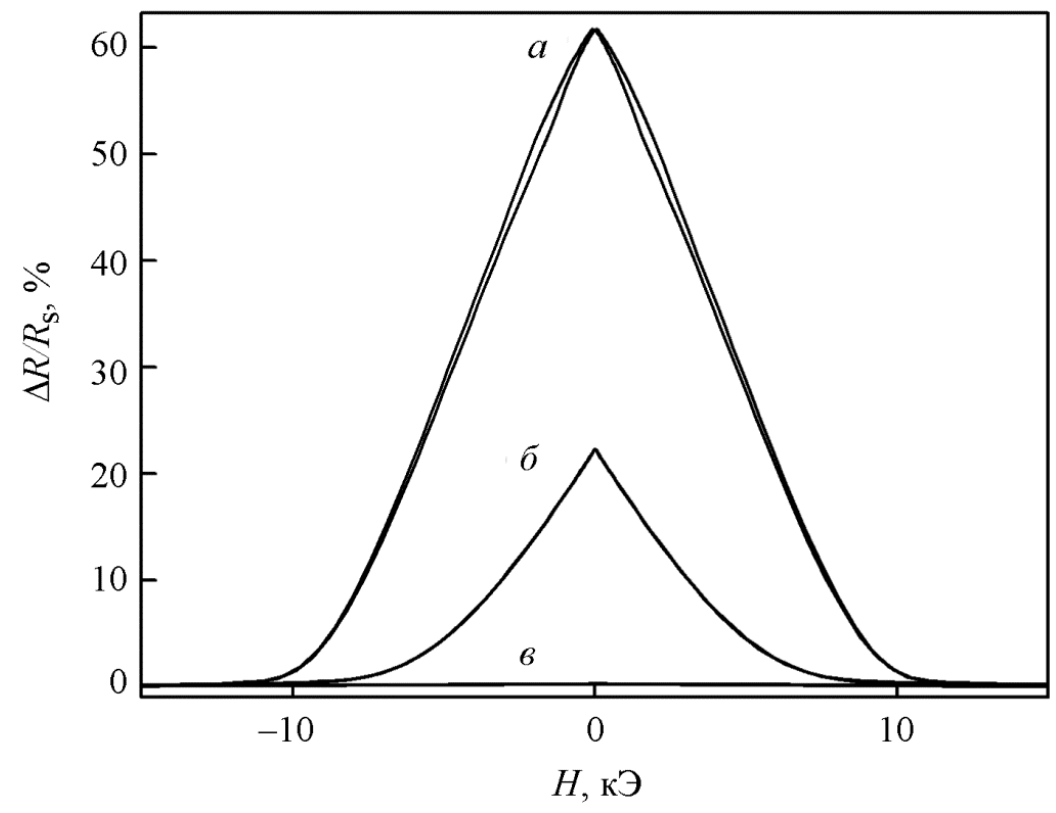

Рис. 1. Зависимость магнитосопротивления сверхрешеток:

Стекло//Ta(5нм)/PyCr(5нм)/[Co ${ }_{90} \mathrm{Fe}_{10}(1,6 \mathrm{Hм}) / \mathrm{Cu}(0,95 \mathrm{Hм}]_{12} / \mathrm{Ta}(5 \mathrm{Hм})$ от температуры отжига: после напыления $(a)$; отжиг при $400{ }^{\circ} \mathrm{C}(\sigma) ; 450{ }^{\circ} \mathrm{C}($ (в) - 1 ч

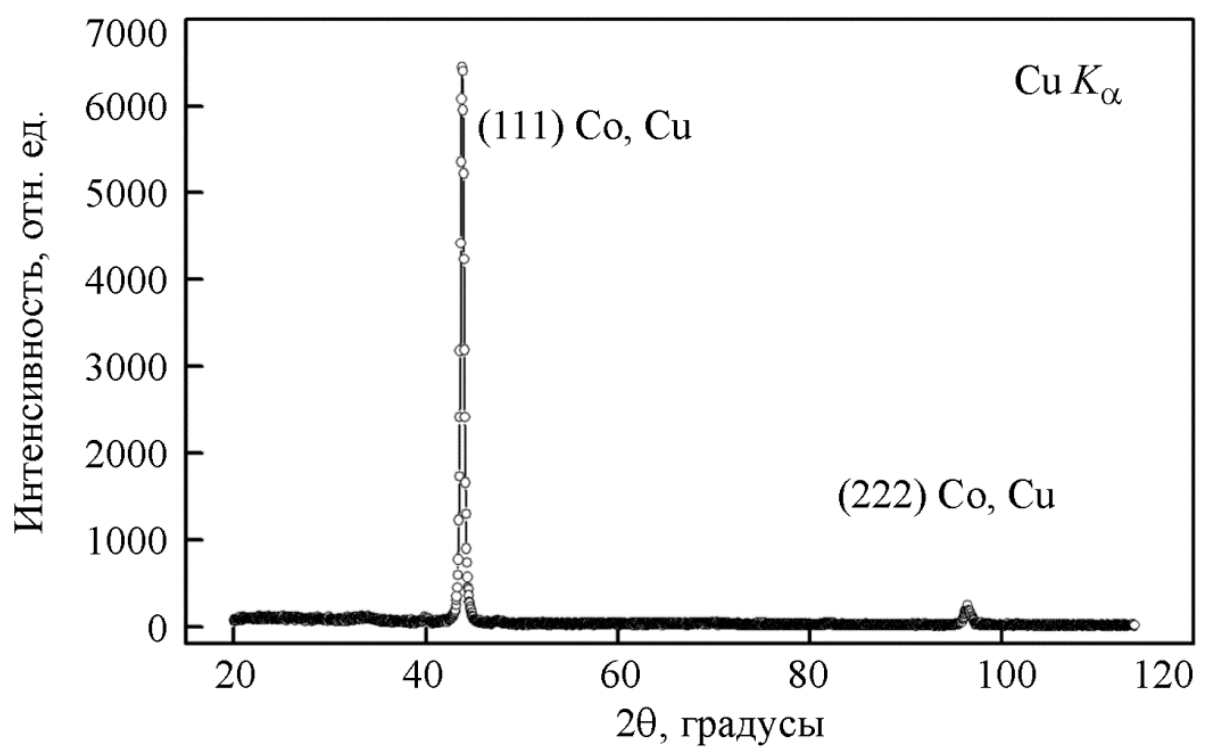

Рис. 2. Рентгеновская дифрактограмма образца:

Стекло//Ta(5нм)/PyCr(5нм)//[Cо ${ }_{90} \mathrm{Fe}_{10}(1,6 \mathrm{Hм}) / \mathrm{Cu}(0,95 \mathrm{Hм}]_{12} / \mathrm{Ta}(5 \mathrm{Hм})$ после напыления

For citation: Chuprakov S. A., Blinov I. V., Milyaev M. A., Popov V. V. Effect of heat treatment on the state of interlayer interfaces and magnetoresistive properties of $\mathrm{Co}_{90} \mathrm{Fe}_{10} / \mathrm{Cu}$ superlattices // Diagnostics, Resource and Mechanics of materials and structures. - 2018. - Iss. 4. - P. 34-41. - DOI: 10.17804/2410-9908.2018.4.034-041. 
На рис. 2 представлена рентгеновская дифрактограмма образца после напыления. На дифрактограмме присутствует набор линий, отвечающая за отражение от плоскостей $\{111\}$, а также кратный рефлекс (222). Таким образом, в исследуемом образце формируется аксиальная текстура с осью $<111>$, перпендикулярной плоскости подложки.

Запись спектров ЯМР была произведена в соответствии с использованной методикой. На рис. 3. представлены примеры моделирования спектров, где маркерами в виде окружности обозначены экспериментальные данные, штриховой линией - Гауссовые пики, каждый из которых соответствует определенному типу ближайшего окружения ядра-зонда, сплошной линией - итоговый смоделированный спектр. В ходе обработки экспериментальных данных выполнялась нормирование интенсивности спинового эха на единицу. Если ядро-зонд находится в объеме слоя Со, тогда в ближайшем окружении все возможные 12 позиций будут заняты атомами Со, в результате чего сверхтонкое поле на ядре-зонде составит примерно $B=21,8 \mathrm{~T}$, резонансная частота - 217,4 МГц [9]. Согласно ранее разработанной модели [11-13], замещение одного атома кобальта одним атомом меди в ближайшем окружении ядра-зонда приведет к уменьшению сверхтонкого поля на ядре-зонде и соответственно к уменьшению резонансной частоты примерно на 18 МГц. При наличии текстуры $<111>$ ядро-зонд в совершенной межслойной границе $\mathrm{Co} / \mathrm{Cu}$ имеет в ближайшем окружении 9 атомов Со и 3 атома $\mathrm{Cu}$ и резонансную частоту примерно на 168 МГц. Поскольку в настоящей работе исследуются сверхрешетки с ферромагнитным слоем $\mathrm{Co}_{90} \mathrm{Fe}_{10}$, то необходимо учесть конфигурации ближайшего окружения с учетом атомов железа. В случае ГЦК-решетки, при замещении одного атома кобальта одним атомом железа в ближайшем окружении ядра-зонда происходит увеличение резонасной частоты на 8 МГц [14].

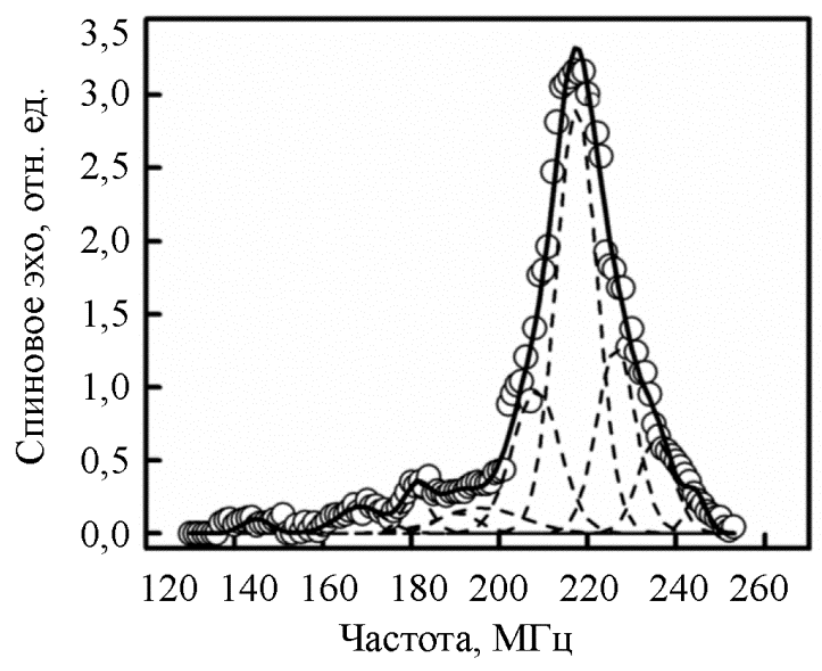

$a$

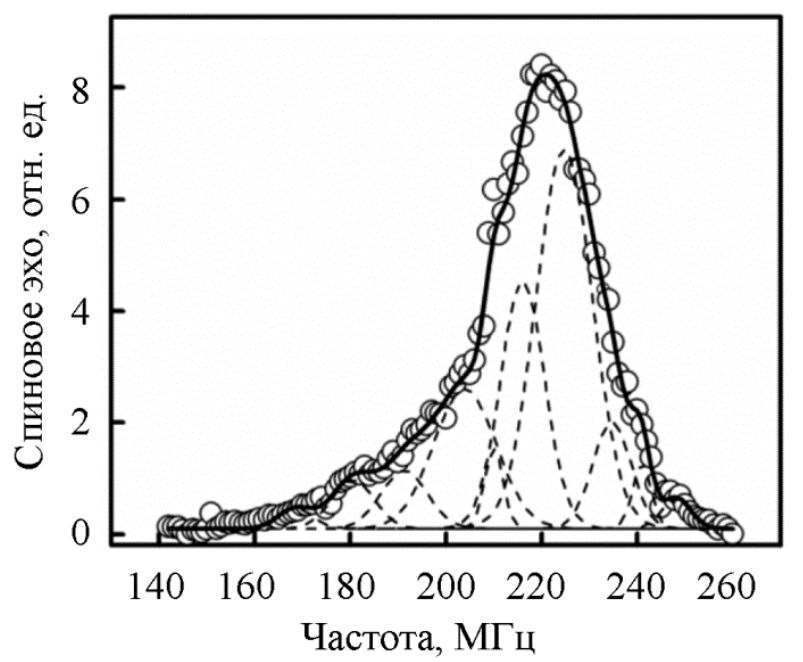

$\sigma$

Рис. 3. Спектры ЯМР для образца:

Стекло//Ta(5нм)/PyCr(5нм)/[CoFe(1,6нм)/Cu(0,95нм $]_{12} / \mathrm{Ta}(5$ нм) после напыления $(a)$ и после отжига при $450{ }^{\circ} \mathrm{C}(б)$, длительностью 1 ч

Для удобства описания спектра выбраны следующие обозначения: $I_{12 / 0 / 0}$ (ближайшее окружения ядра зонда: 12 атомов $\mathrm{Co}, 0-\mathrm{Cu}, 0-\mathrm{Fe}$ ), $I_{10 / 1 / 1}$ (ближайшее окружение ядра-зонда: 10 атомов $\mathrm{Co}, 1 \mathrm{Cu}, 1 \mathrm{Fe})$. При моделировании варьировались следующие параметры Гауссовых пиков: положение (частота), интенсивность, ширина линии. Для проверки полученных результатов моделирование проводилось с различными наборами начальных условий.

Гауссовский пик $I_{12 / 0 / 0}$ соответствует ядру-зонду, находящемуся в объеме слоя Со, и соответственно имеет в ближайшем окружении 12 атомов Со. Пик $I_{9 / 3 / 0}$ на частоте 168 МГц сформирован ядрами атомов Со высокосовершенного интерфейса $\mathrm{CoFe} / \mathrm{Cu}$, совпадающего с плотноупакованной плоскостью <111> ГЦК-решетки, когда каждый атом Со имеет в бли- 
жайшем окружении три атома $\mathrm{Cu}$. Также высокосовершенной границей мы считаем $I_{9 / 2 / 1}$, как предыдущий тип границы, но один атом $\mathrm{Cu}$ замещен $\mathrm{Fe}$.

Долю высокосовершенных границ можно определить через отношение суммы $I_{9 / 3 / 0}$ и $I_{9 / 2 / 1}$ К сумме интенсивностей остальных спектральных линий, кроме характеризующих ядро-зонд внутри слоя CoFe: $\frac{I_{9 / 3 / 0}+I_{9 / 2 / 1}}{\text { interfaces }}$. Полученная зависимость доли высокосовершенных границ от температуры отжига приведена в табл. 1. Доля высокосовершенных границ после напыления составляет $34 \%$. При отжиге доля высокосовершенных границ уменьшается с $24 \%\left(400{ }^{\circ} \mathrm{C}\right)$ до $6 \%\left(450{ }^{\circ} \mathrm{C}\right)$.

Таблица 1 - Доля высокосовершенных границ в зависимости от температуры отжига

\begin{tabular}{|c|c|}
\hline $\begin{array}{c}\text { Температура } \\
\text { отжига, }{ }^{\circ} \mathrm{C}\end{array}$ & $\begin{array}{c}\text { Доля высокосовершенных } \\
\text { границ, } \%\end{array}$ \\
\hline Без отжига & 34 \\
\hline 400 & 24 \\
\hline 450 & 6 \\
\hline
\end{tabular}

Состояние межслойных границ можно также характеризовать долей атомов Со, локализованных в интерфейсах. Долю таких атомов можно определить как отношение суммарной интенсивности пиков, сформированных атомами, находящимися в интерфейсах, к суммарной интенсивности всех пиков: $\frac{\sum \text { interfaces }}{\sum \text { spectrum }}$. Доля атомов кобальта в интерфейсе для образца после напыления составляет $21 \%$. При отжиге доля таких атомов увеличивается от $30 \%$ $\left(400{ }^{\circ} \mathrm{C}\right)$ до $44 \%\left(450{ }^{\circ} \mathrm{C}\right)$.

Таблица 2 - Доля атомов Со в интерфейсе в зависимости от температуры отжига

\begin{tabular}{|c|c|}
\hline $\begin{array}{c}\text { Температура } \\
\text { отжига, }{ }^{\circ} \mathrm{C}\end{array}$ & $\begin{array}{c}\text { Доля атомов Со } \\
\text { в интерфейсе, } \%\end{array}$ \\
\hline Без отжига & 21 \\
\hline 400 & 30 \\
\hline 450 & 44 \\
\hline
\end{tabular}

Из табличных данных видно, что в результате отжига доля атомов, участвующих в формировании интерфейсов возрастает от $21 \%$ (для образца после напыления) до 44 \% для образца после отжига при $450{ }^{\circ} \mathrm{C}, 1$ ч. Это свидетельствует о том, что при отжиге происходит размытие межслойных границ и, как следствие, уменьшение магнитосопротивления.

\section{4. Заключение}

Методами ядерного магнитного резонанса и рентгеновской дифракции исследованы структурные особенности сверхрешеток Стекло//Тa(5нм)/PyCr(5нм)/[Cо ${ }_{90} \mathrm{Fe}_{10}(1,6 \mathrm{Hм}) / \mathrm{Cu}(0,95 \mathrm{Hм}]_{12} /$ $/ \mathrm{Ta}(5$ нм $)$ и влияние состояния интерфейсов $\mathrm{Co} / \mathrm{Cu}$ на их магниторезистивные свойства. Показано, что с увеличением температуры отжига происходит уменьшение магнитосопротивления, что обусловлено изменением состояния интерфейсов: уменьшается доля высокосовершенных интерфейсов, возрастает число атомов кобальта, участвующих в формировании межслойных границ. 


\section{Благодарности}

Рентгенографические исследования проведены на дифрактометре Eтругеan PANalytical Series 2 ЦКП ИФМ УрО РАН.

Работа выполнена в рамках государственного задания по теме «Спин» №AAAA-A18118020290104-2 и проекту №18-10-2-37 Программь УрО РАН.

\section{Список литературы}

1. Wetzig K., Schneider C. M. Metal Based Thin Films for Electronics. - Weinheim : WILEYVCH GmbH \& Co., KGaA, 2003. - 388 p. - DOI: 10.1002/3527602534.

2. Mallinson J. C. Magnetoresistive and spin valve heads. Fundamentals and applications. 2-nd ed. - San Diego : ACADEMIC PRESS, 2002. - 179 p.

3. Kataoka N., Saito K., Fujimori H. Magnetoresistance of Co-X/Cu multilayers // J. Magn. Magn. Mater. - 1993. - Vol. 121. - P. 383-385. - DOI: 10.1016/0304-8853(93)91226-W.

4. Saito Y., Hashimoto S., Inomata K. Giant magnetoresistance in $\mathrm{Co} / \mathrm{Cu}, \mathrm{Co} 9 \mathrm{Fe} / \mathrm{Cu}$, and Co7.5Fe2.5/Cu multilayers // IEEE Transactions on Magnetics. - 1992. - Vol. 28, no. 23. P. 2751-2753. - DOI:10.1109/20.179617.

5. Wang D., Anderson J., Daughton J. M. Thermally stable, low saturation field, low hysteresis, high GMR CoFe/Cu multilayers // IEEE Transactions on Magnetics. - 1997. - Vol. 33, no. 5. P. 3520-3522. - DOI: 10.1109/20.619484.

6. Kuch W., Marley A. C., Parkin S. S. P. Seeded epitaxy of Co90Fe10/Cu multilayers on $\mathrm{MgO}(001)$ :Influence of Fe seed layer thickness // J. Appl. Phys. - 1998. - Vol. 83, no. 9. P. 4709-4713. - DOI:10.1063/1.367259.

7. Magnetoresistance of Co_X/Cu (X=Fe, Ni) multilayers / H. Kano, Y. Iwasaki, K. Hayashi, K. Aso // J. Magn. Magn. Mater. - 1993. - Vol. 126. - P. 445-447. - DOI: 10.1016/03048853(93)90651-H.

8. Ustinov V. V., Milyaev M. A., Naumova L. I. Giant Magnetoresistance of Metallic Exchange-Coupled Multilayers and Spin Valves // Physics of Metals and Metallography. - 2017. Vol. 118. - P. 1300-1359. - DOI:10.1134/S0031918X17130038.

9. Thomson T., Riedi P. C., Greig D. Interfacial quality and giant magnetoresistance in MBEgrown $\mathrm{Co} / \mathrm{Cu}(111)$ superlattices // Phys. Rev. B. - 1994. - Vol. 50, no. 14. - P. 10319-10322. DOI: 10.1103/PhysRevB.50.10319.

10. ${ }^{59} \mathrm{Co}$ nuclear magnetic resonance studies of interfacial defects in molecular beam epitaxy grown $\mathrm{Co} / \mathrm{Cu}(111)$ multilayer / T. Thomson, P. C. Riedi, K. P. Wellock, B. J. Hickey // J. Appl. Phys. -1995. - Vol. 81, no. 8. - P. 4469-4471. - DOI: 10.1063/1.364980.

11. Interface Structure and Magnetoresistance Studies of $[\mathrm{Co} / \mathrm{Cu}] n$ Superlattices by Means of NMR and TEM / S. A. Chuprakov, T. P. Krinitsin., N. S.Bannikova, I. V. Blinov, S. V. Verkhovskii, M. A. Milyaev, V. V. Popov, V. V. Ustinov // Solid State Phenomena. - 2014. - Vol. 215. P. 358-364. - DOI: 10.4028/www.scientific.net/SSP.215.358.

12. Investigation of interfaces of multilayer $\mathrm{Co} / \mathrm{Cu}$ structures using the method of nuclear magnetic resonance / S. A. Chuprakov, N. S. Bannikova, I. V. Blinov, T. P. Krinitsina, M. A. Milyaev, V. V. Popov, V. V. Ustinov // Physics of Metals and Metallography. - 2015. - Vol. 116. P. 136-140. - DOI: 10.1134/S0031918X15020040.

13. Study of the structure of interlayer boundaries in $[\mathrm{Co} / \mathrm{Cu}] 10$ superlattices by methods of NMR and X-ray reflectometry / S. A. Chuprakov, N. S. Bannikova, I. V. Blinov, T. P. Krinitsina, M. A. Milyaev, V. V. Popov, V. V. Ustinov // Physics of Metals and Metallography. - 2016. Vol. 117. - P. 1192-1197. - DOI: 10.1134/S0031918X16120048.

14. Thomson T., Riedi P. C. NMR Studies of Sputtered CoFe Alloy Thin Films // IEEE Trans Magn. - 1998. - Vol. 34, no. 4. - P. 1045-1047. - DOI: 10.1109/20.706352. 